\title{
Redefiniendo el concepto del remanente urbano: una mirada en la ciudad de Mexicali, Baja California
}

\author{
Redefining the urban concept of the lost spaces: a look at the city of Mexicali, \\ Baja California
}

Recibido: mayo 2020

Aceptado: febrero 2021

\section{Resumen}

Hablar sobre los remanentes urbanos nos lleva a reflexionar acerca de diversos espacios perdidos que cotidianamente observamos en el paisaje urbano de una ciudad, básicamente este fenómeno tiene que ver con aquellos derechos de vías que van quedando olvidados al costado de avenidas, vías férreas, ríos ó incluso canales, también hablamos de aquellos espacios bajo puentes o distribuidores viales, así como de camellones o guarniciones inconclusas; en general son aquellos fragmentos de ciudad que gradualmente van resultando como sobrantes de un proyecto de gran escala así como de una producción urbana desordenada y especialmente descuidada en cuanto a brindar atención (preventivamente desde la ejecución del proyecto urbano) ó solución (correctivamente desde los planes de desarrollo municipales). En la actualidad los remanentes urbanos se presentan como una forma de anti-espacio urbano y puntualmente deben ser comprendidos como una tipología especifica que pertenece al ámbito de los espacios residuales, y en ese sentido el presente trabajo de investigación se pretendió llevar más allá de un conjunto de reflexiones teóricas debido a que también se expone un ejercicio práctico, el cual estuvo enfocado en realizar un levantamiento de casos de estudio en la ciudad de Mexicali y donde fue posible detectar un total de 123 casos prácticos que fueron identificados dentro del área urbana consolidada de esta ciudad.

\section{Palabras Claves:}

remanentes urbanos; espacios residuales; anti-espacio urbano

\author{
Claudio Curzio ${ }^{1}$ \\ Tonahtiuc Moreno Codina ${ }^{2}$
}

\begin{abstract}
Talk about the urban remnants leads us to analyze about various types of lost spaces that we can see on a daily basis in the urban landscape of a city, basically this phenomenon has been related with those rights of way that are being forgotten along the avenues, railways, rivers or even canals, we also speak of those spaces under bridges or road distributors, as well as unfinished sidewalks or central medians; so this type of lost spaces are those fragments of the city that gradually turn out to be leftovers from a large-scale project as well as from a disorderly urban production and especially neglected in terms of the proper caution (preventively from the execution of the urban project) or the proper solution (correctively from the plans of municipal development). Currently, urban remnants are presented as a form of urban anti-space and should be specifically understood as a specific typology that belongs to the field of the residual spaces, in this sense, the current research work is more than a set of theoretical reflections because a practical exercise is also exposed, which was focused on review and analyses some specific points in the city of Mexicali and where it was possible to detect a total of 123 practical cases that were identified within the consolidated urban area of this city.
\end{abstract}

\section{Keywords:}

urban lost spaces; residual spaces; urban anti-space

\footnotetext{
1 Nacionalidad: mexicano; adscripción: Universidad Autónoma de Aguascalientes; correo: claudio7curzio@icloud.com

2 Nacionalidad: mexicano; adscripción: Universidad Autónoma de Aguascalientes; correo: tonahtiuc@hotmail.com
} 


\section{INTRODUCCIÓN}

En la actualidad los remanentes urbanos se presentan como un fenómeno que resulta recurrente y paralelamente también se encuentra en constante crecimiento, especialmente esto se acentúa en aquellas ciudades que se encuentran en pleno proceso de desarrollo; inclusive pareciera que la vocación de dicho desarrollo urbano se va danto en el contexto de una agenda muy apretada que esta manejada por los intereses que dictan el ordenamiento territorial y al mismo tiempo esto se va dando de una forma tan acelerada que frecuentemente se observa una carencia para brindar soluciones finas y específicas en ámbitos relativos al diseño del paisaje urbano. En ese sentido la complejidad de poder atender esos temas radica primordialmente en la lógica proyectual que rige actualmente la transformación y evolución de la ciudad; básicamente dicha lógica ha quedado enmarcada bajo los criterios de racionalidad análogos a los de la ciencia y la técnica. Con ello, arquitectos y urbanistas han volcado su imaginar en pretender ordenar el territorio teniendo como meta máxima aquel progreso ilimitado que enalteció por siglos el pensamiento moderno.

El surgimiento de los remanentes urbanos es en parte un claro reflejo de ese pensamiento en el que el hombre queda relegado a jugar papeles secundarios dentro de su propia ciudad, diversos factores políticos, económicos y culturales han ido banalizando el contenido humano de la arquitectura urbana y de los criterios que deben regir el ordenamiento territorial.

Básicamente desde la primera mitad del siglo XX (paralelamente con la llegada del automóvil) ya era posible observar el surgimiento de una crisis urbana, derivada entre otras cosas de esa ruptura en la confianza ciega y desbordante hacia el progreso científico-técnico, la cual ha venido ensombreciendo la producción arquitectónica en las diversas escalas que ésta se presenta; ya que si bien es cierto que la creación de ciudad siempre ha respondido a un único llamado de racionalidad este hecho no precisamente ha representado ser lo correcto. Probablemente el primer gran parteaguas se dio en 1933 con la carta de Atenas en donde claramente ya se ponía de manifiesto la crisis que empezaban a experimentar algunas de las grandes urbes de la época. Específicamente en dicha carta se denunciaba la caótica situación y la irracionalidad de amplios sectores urbanos de aquella época. Concretamente se hace referencia a las densidades excesivas, déficit de servicios, escasa calidad ambiental, insalubridad, reparto arbitrario de usos del suelo, estrechez de las calles, tipologías residenciales insufribles y globalmente se descalifica todo valor positivo a las ciudades contemporáneas.

Sin embargo la carta de Atenas se quedó rápidamente en un elemento obsoleto que únicamente sirvió para comunicar buenas intenciones y enfatizar el hecho de que existía una crisis a nivel urbano, pero las medidas tomadas al respecto por gobiernos é instituciones en corto y largo plazo no resultaron congruentes a la problemática, sino que por el contrario, muchos especialistas como Peter Hall (1996) se atrevieron a señalar que algunos de los planteamientos urbanos de las últimas décadas han resultado "autodestructivos" debido a que se rigen principalmente por los intereses económicos inmediatos. En los años setentas, el urbanismo cambio totalmente y en los ochentas parecía abocado a la autodestrucción. Daba la sensación de que la planificación convencional y el uso de planes y normas para reglamentar el uso de suelo habían caído en total descrédito. En lugar de regular el crecimiento urbano, el urbanista se había dedicado a fomentarlo con todos los recursos que tenía a su alcance. La idea que predominaba era de que la ciudad era una máquina de crear riqueza y que la función del urbanismo era engrasar esa maquinaria. (Hall, 1996, p. 354)

De tal forma que desde la segunda mitad del siglo XX y hasta la fecha, en gran parte de las ciudades contemporáneas es posible percibir que las redes de infraestructura urbana (principalmente aquellas destinadas al transporte) son en gran medida los principales ejes rectores en la morfología de las ciudades y en consecuencia rigen el sentido del crecimiento territorial y del ordenamiento espacial. Esta situación se ha logrado ver reflejada en cuanto a que la discontinuidad y la fragmentación son características que gradualmente se han vuelto más recurrentes, básicamente se han venido transformado la ciudad compacta en una ciudad dispersa (un conjunto de fragmentos urbanos aislados). De tal modo que la ciudad contemporánea ha configurado una nueva estructura desde la necesidad de atender las nuevas demandas de movilidad mediante la construcción de nuevas redes tanto viarias como ferroviarias. La influencia de la movilidad es una 
de las claves de esta dispersión urbana (Berruete, 2017, p. 117). En forma muy particular, el automóvil es el elemento que ha adquirido un valor preponderante desde la segunda mitad del siglo XX hasta nuestros días, pese a ser un medio de transporte privado (y en muchos casos no incluyente) las calles, avenidas, carreteras y autopistas, se han consolidado como el principal elemento rector en el ordenamiento territorial de la urbe contemporánea.

Sin embargo, vale la pena mencionar que previo a que se introdujera masivamente el uso del automóvil, las ciudades estaban concebidas de un modo distinto, generalmente existía un núcleo primitivo cuya superficie estuvo delimitada durante mucho tiempo por la necesidad de efectuar los desplazamientos a pie ante la inexistencia de sistema de transporte colectivo (Zarate Martin, 2003, p. 120). De este modo caminar por la ciudad dejó de ser una opción para comunicarse entre los barrios y en su lugar, usar el transporte público o un vehículo particular se hizo casi obligatorio (Díaz Cruz, 2015, p. 68). Ese incremento brutal de la utilización del automóvil en la ciudad, así alimentado, hace a los técnicos municipales plantearse la necesidad de ensanchar o duplicar las vías en altura, de acomodar al automóvil lo que no se pensó para él; los monstruosos pasos a desnivel de acero y hormigón destrozan el paisaje urbano, atraen a más coches hacia el centro y crean más atascos de los que se pretendían solucionar; el presunto remedio acelera el curso de la enfermedad, el ahogo y la asfixia de la ciudad (García Bellido \& Gonzalez Tamarit, 1979, p. 84). Paralelamente, en el caso específico de Mexicali también es posible observar que coincidentemente desde la segunda mitad del siglo XX se ha venido acelerando un proceso de metamorfosis urbana. En primer término el crecimiento demográfico es un primer reflejo de la evolución que ha venido experimentando esta ciudad; concretamente en el año de 1960 Mexicali contaba con una población de 174,540 habitantes y en un lapso de cinco décadas este número se elevó significativamente hasta llegar a los 936,826 habitantes en el año de 2010 (INEGI, 2010).

Paralelamente la condición de ciudad fronteriza también gradualmente ha ido condicionando que la metamorfosis urbana en Mexicali vaya teniendo una íntima relación con el crecimiento de la diversidad en prestaciones de servicios y fuentes de trabajo; por ejemplo la producción agrícola dejó de ser la actividad económica principal en la región, viéndose remplazada de forma paulatina por la industria manufacturera y la prestación de servicios. El acelerado crecimiento concuerda con la intensificación de la industria en la región fronteriza, de tal forma que de 1980 a 1998 el número de empresas maquiladoras establecidas en el municipio aumentó un $227 \%$, al pasar de 79 a 180 (Vizcarra, 2019, p. 313 y 314). De esta manera durante los últimos cincuenta o sesenta años Mexicali ha experimentado un crecimiento socio-económico que ha traído como consecuencia una infraestructura urbana de mayor complejidad (construcción de pasos a desnivel, puentes, distribuidores y ejes viales, así como el ensanchamiento de avenidas).

Sin embargo, todo este crecimiento se ha dado de una forma desequilibrada y sin contrapesos debido a que Mexicali carece de programas parciales de desarrollo urbano, que conduzcan el crecimiento de zonas específicas de la ciudad. De igual modo, el equipamiento urbano es deficitario enáreas verdes, recreativas, culturales y deportivas, faltan áreas de estacionamiento en el centro de la ciudad, así como la ocupación irregular de la vía pública por invasión de comercios fijos, semi-fijos y ambulantes; existe también una proliferación de asentamientos humanos irregulares por diferentes puntos de la ciudad, pues hay insuficiencia de suelo y vivienda, consecuentemente originan deterioro en algunos aspectos como el espacial y el ambiental. Además, en la zona urbana existe una gran cantidad de predios baldíos y construcciones abandonadas, lo que genera una subutilización del espacio y de la capacidad instalada de los servicios (Padilla y Sotelo \& Juárez, 2000, p. 101). En resumen, no hace falta ser un especialista en materia urbana para poder asegurar que Mexicali y muchas otras grandes metrópolis han crecido desmedidamente hasta el punto de encontrarse con un desbordamiento de sus estructuras urbanas, muchas de las ciudades de mediana escala ó provincias no han terminado por redefinir su postura ante la nueva situación económica mundial y en contraparte el ámbito rural se encuentra en un profundo y cada vez más angustiante rezago en un sector muy importante de los países Latinoamericanos.

De tal forma que el problema del desbordamiento de la escala urbana es sin duda un ámbito que ha repercutido directamente en 
una falta de cohesión al momento de pretender realizar una integración del tejido urbano, y en ese sentido entrando al tema de los remanentes urbanos, es posible asegurar que en cuanto mayor sea la escala de producción urbana, mayor será la probabilidad de que el diseño en su conjunto arroje fragmentos remanentes ó "sobrantes" ya que existe una menor atención por el manejo del detalle urbano; de cierta forma es válida la expresión de que "lo pequeño es más urbano". Los monumentales ejes principales que aparecen en los proyectos más característicos contribuyen a crear un modelo unificado, mientras que la gran escala perjudica al detalle y aplasta al individuo. (Rosenau, 1986, p. 177). En síntesis, es posible señalar que ese objetivo puramente técnico que se ha venido dando en la producción urbana, se ha sumado a un desbordamiento en la escala del proyecto han llevado a que en la segunda mitad del siglo XX la producción urbana en su conjunto arroje una infinidad de fragmentos, espacios sin uso, hablamos de remanentes urbanos...

\section{MARCO TEÓRICO}

\section{Definición, clasificación y principales} características:

Son diversos los estudios que se han realizado en relación a temas vinculados con el anti-espacio, normalmente cada uno de estos autores ha acuñado un término específico para referirse a fenómenos urbanos muy concretos que pretenden explicar, principalmente algunos de los conceptos más usados son: espacios muertos, intersticios, espacios residuales, vacíos urbanos, espacios abandonados, espacios indefinidos, espacios indeterminados, espacios perdidos, etc. "Se debe tener mucho cuidado con la terminología, que se asocia con aquellos espacios urbanos no utilizados. Cuando se trata de elementos construidos, por ejemplo, el término "vacante" se refiere a edificios abandonados. En contraste con los terrenos vacíos que nunca tuvieron ninguna forma de ocupación. Algunas definiciones de espacio urbano no utilizado enfatizan el vacío del terreno, en comparación con el entorno construido circundante, el hecho de que no estén ocupados por personas ni construcciones e infraestructura". (Nefs, 2006, p. 49) Coincidentemente, en el caso específico de los remanentes urbanos aún sigue predominando una falta de claridad para definir y exponer la tipología y las características de dicho fenómeno.
Figura 1. Diagrama que muestra la clasificación para el estudio del anti-espacio

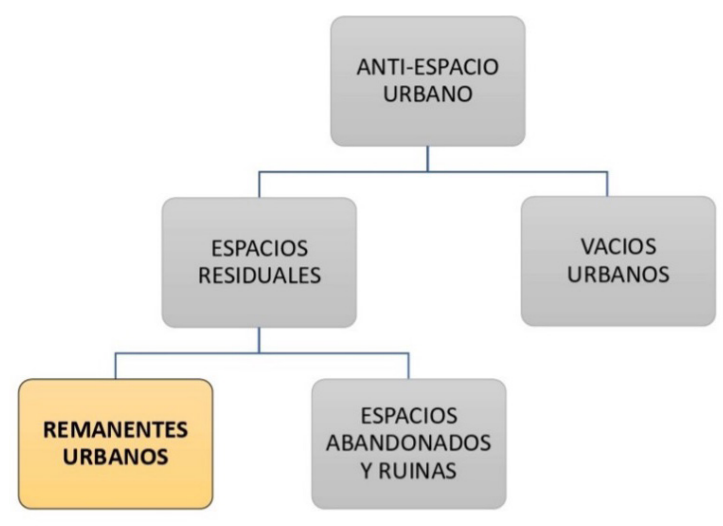

Fuente: Elaborado por los autores

En primer término, para lograr comprender de forma amplia y concisa el significado del remanente urbano es preciso remontarse a analizar el significado del termino "residual", es preciso remontarnos a analizar el significado del término como tal (desde el punto de vista etimológico) podemos encontrar que el origen se deriva de la raíz latina «residûum», cuyo significado textual es el siguiente: «1. Parte ó porción que queda de un todo 11 2. Lo que resulta de la descomposición ó destrucción de una cosa. 11 3. Alg. y Arit. Resultado de la operación de restar» ${ }^{3}$.

Tomando como base el significado nominal del término "residual", es posible afirmar que los remanentes urbanos pueden ser comprendidos como aquella parte o porción espacial que se derivan de un proyecto urbano de mayor escala, se trata de fragmentos urbanos que no cumplen con una función definida pese a encontrarse fisicamente delimitados e inmersos en un entramado urbano. En palabras de Francisco Berruete, se trata de aquellos tipos que generan espacios atrapados dentro de los nudos de comunicación se pueden considerar como "islas" otro caso que se puede observar, es el que aparece en los límites de infraestructuras, básicamente en bordes de las nuevas autopistas que generan este tipo de retazos. (Berruete, 2015, p. 189). De tal modo que, de manera más específica, dentro de esta categoría de anti-espacio podemos encontrar 3 diferentes tipologías (siguiente página):

\footnotetext{
${ }^{3}$ Fuente: Diccionario de la lengua española (www. http://dle.rae. es/?id=LxCxNlf)
} 
Figura 2. Tipología que presentan los remanentes urbanos

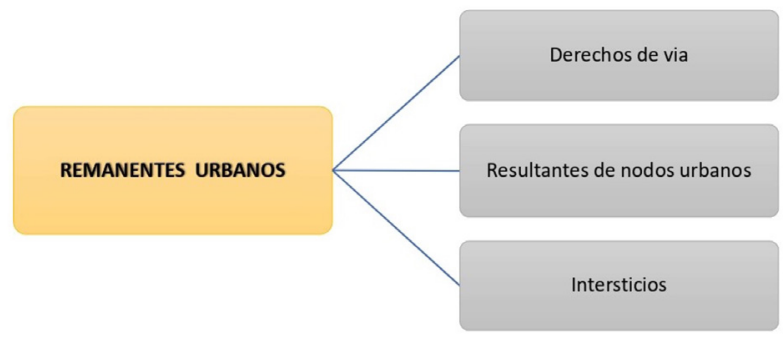

Fuente: Elaborado por los autores

Básicamente en esta categoría de antiespacio podemos identificar que las diversas variables tienen como un común denominador el hecho de que se trata de espacios que fueron quedando (sobrando) de diversos proyectos de infraestructura urbana, como por ejemplo uno de los casos más frecuentes es el de los derechos de vía que surgen después del trazado de vías de comunicación (vehiculares o férreas), así como también es frecuente observar derechos de vía que corresponden a infraestructura de diversas instalaciones (primordialmente eléctricas y de telecomunicaciones).

Figura 3. Ejemplo de remanente urbano, derecho de vía (férrea) en Mexicali, B.C

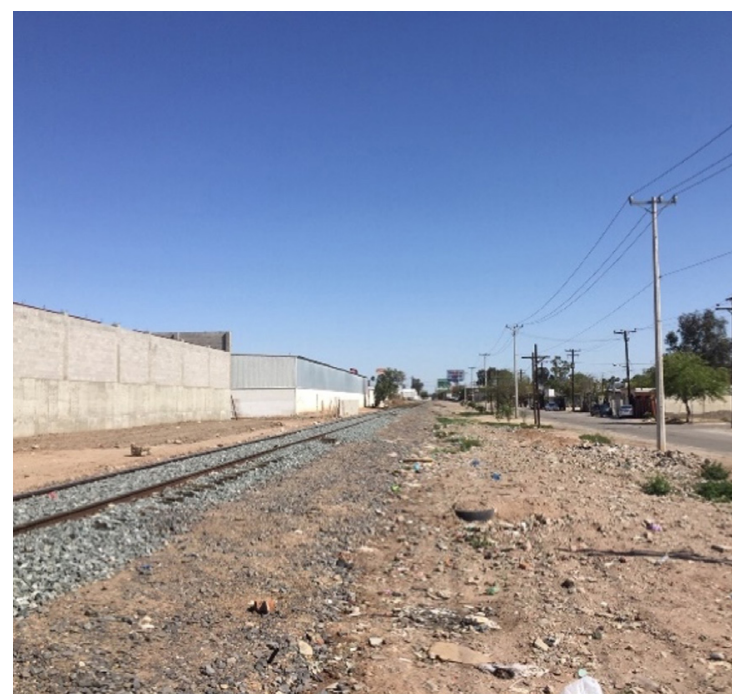

Fuente: Fotografía por los autores

Por otra parte, existe el caso de ciertos sobrantes derivados de la misma producción urbana que van quedado aislados dentro de la estructura urbana de una ciudad; específicamente se trata de espacios remanentes que de igual manera también surgen como un residuo que es resultado de proyectos urbanos de gran escala y están caracterizados por no cumplir con ninguna función ni aportan nada a los habitantes de la ciudad donde se localicen. Dentro de esta categoría podemos ubicar los espacios sobrantes que van quedando sin atenderse debajo de puentes vehiculares o también por ejemplo se encuentra el caso de los remanentes localizados en los nodos de cruce de vialidades.

Figura 4. Ejemplo de remanente urbano, se trata de la resultante de un nodo urbano localizado en EI Paso, TX

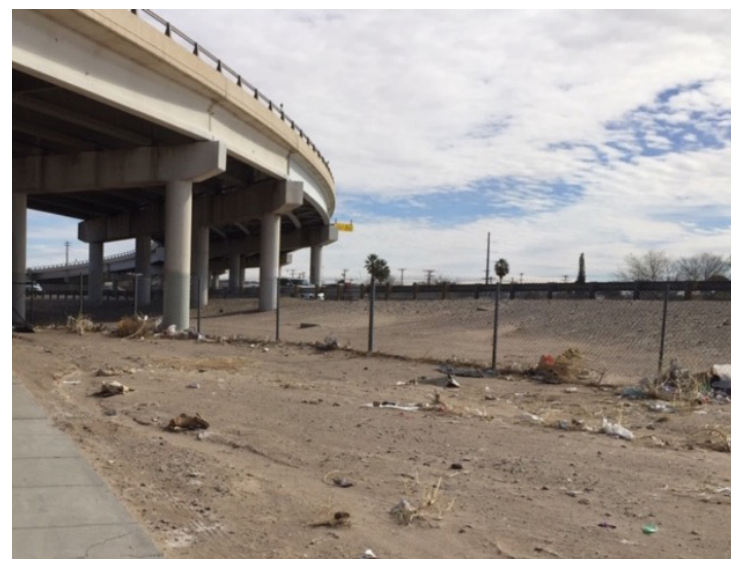

Fuente: Fotografia por los autores

Coincidentemente con el surgimiento de esta serie de sobrantes derivados de la producción urbana, también destaca la presencia de los subproductos urbanos, los cuales básicamente se trata de una serie de espacios que también comparten el mismo origen sin embargo la diferencia radica en que los mismos habitantes se han encargado de brindar un uso informal o temporal, normalmente esa ocupación es caracterizada por manifestarse de una forma espontánea y desordenada. De manera específica el término del subproducto urbano surge del siguiente razonamiento: "Un residuo, que no se produce intencionalmente, pero para el que el proceso de producción ha sido adaptado de forma que sea reciclable -in situ-, no es un residuo sino un subproducto". (Hannequart, 1993)

Este tipo de subproductos urbanos es común que se observen justamente en esos espacios debajo de los puentes vehiculares en donde han dejado de ser lugares desolados para convertirse en puntos donde se establecen comercios informales. (Ver Figura 5 p. 76) 
Figura 5. Ejemplo de subproducto urbano localizado en Ciudad de México

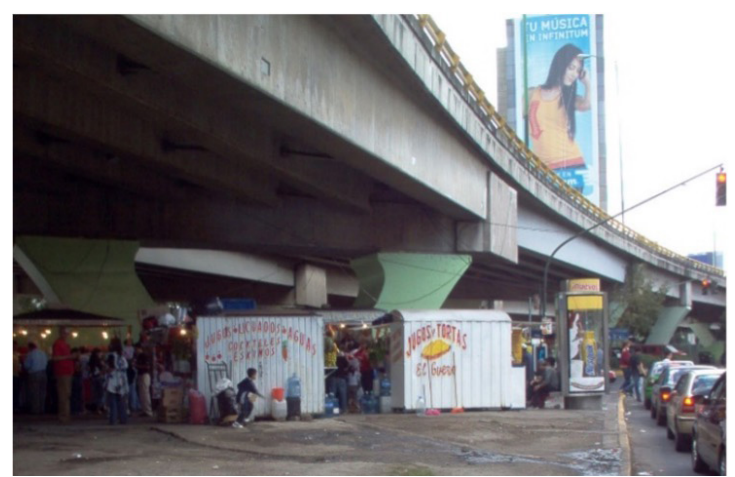

Fuente: Fotografia por los autores

Por otra parte, los remanentes urbanos también pueden manifestarse con la tipología del intersticio ó espacios intermedios ("Interstices - Intermediate spaces"). Es prudente señalar que después de revisar la forma en la que se han venido usando ambos términos es posible deducir que normalmente se están empleando como sinónimos que apuntan a un mismo significado. Sin embargo con el objetivo de comprobar la anterior hipótesis, revise el origen etimológico de la palabra Intersticio, encontrando que dicho termino proviene de la raíz latina interstitium, y cuyo significado nominal es: "Hendidura o espacio, por lo común pequeño, que media entre dos cuerpos o entre dos partes de un mismo cuerpo",4.

Figura 6. Ejemplo de Intersticio localizado en la ciudad de Bogotá, Colombia

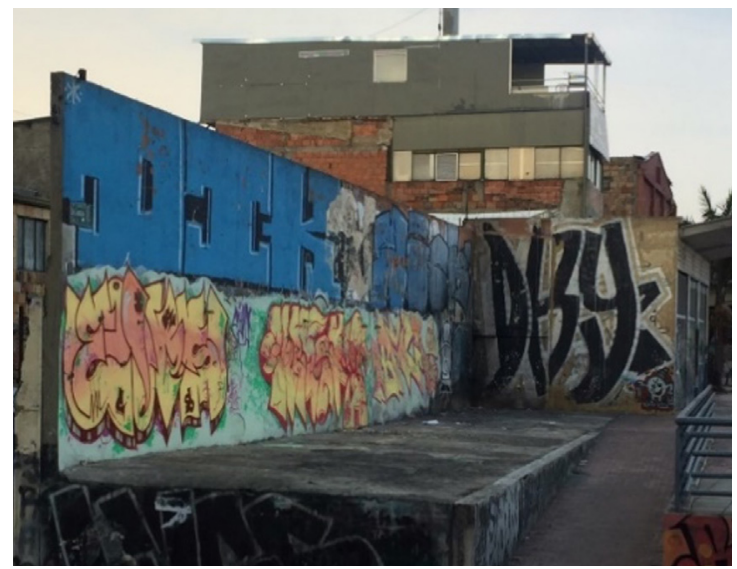

Fuente: Fotografía por los autores

\footnotetext{
${ }^{4}$ Fuente: Diccionario de la lengua española (www. http://dle.rae. es/?id=LxCxNlf)
}

La anterior definición comprueba la equivalencia entre los términos espacios intermedios é intersticios, tratándose en ambos casos de espacios que fueron quedando atrapados entre elementos de mayor escala, entre los edificios inmersos dentro de la urbe, en ese sentido vale la pena mencionar a Doreste (2011) quien también manifiesta como característica relativa a estos fenómenos urbanos el hecho de que se perciben como un elemento con inexistencia desde la mirada del hombre, dicho de otra forma, son espacios que normalmente pasan desapercibidos.

"El intersticio acontece en los lugares de en medio, justo en los márgenes provisionales y anónimos. El espacio entre edificios puede dejar un solar de descuidado bosque caótico dispuesto al residuo. Una especial naturaleza urbana de complicada estética, a la que se le da la espalda. No se tiene en cuenta su existencia, a excepción de algún grafitero casual que ve en ella un soporte libre sobre el cual subrayar sus ansias de identidad". (Doreste, 2011, p. 276). Este mismo concepto también se aborda desde el punto de vista de otro autor, en este caso Giovanni Maciocco (2008) utiliza el termino de espacios intermedios para definir lo que el mismo llama como áreas obsoletas que se encuentran olvidadas en el desarrollo de las ciudades contemporáneas. Este punto de vista resulta interesante porque dentro de la definición que realiza Maccioco profundiza su análisis al mencionar que no se trata de un asunto unidimensional con características meramente físicas (en cuanto a fungir como límites territoriales) sino vas allá al mencionar que se trata de zonas que pese a estar abandonadas representan un interés a nivel cultural y son espacios en los cuales debe predominar el intercambio interdisciplinario para poder superar (rehabilitar) dicho elemento urbano.

"Los espacios intermedios, entonces, entendían no solo o no tanto como el límite zonas en el sentido territorial, sino como zonas de interés cultural e intercambio disciplinario, ya que los intentos de "superarlo" constituían órdenes culturales intentos que solo son posibles en territorios externos a la metrópolis. En el contexto de nuestras metrópolis contemporáneas, las áreas fronterizas, obsoletas, olvidadas por el desarrollo, se presentan así mismas con este carácter". (Maciocco, 2008, p. 137) 
Sintetizando todos los conceptos que están integrados bajo la categoría de los remanentes urbanos, es posible señalar que básicamente hablamos de elementos urbanos que de cierta manera fueron quedando gradualmente olvidados, atrapados y relegados entre el dinamismo de la producción urbana. Tal y como señala (Cléments, 2007, p. 12) este tipo de espacio por lo general procede del principio de Ordenación. En términos generales es posible asegurar que de entrada esta forma de anti-espacio urbano puede ser comprendida desde el punto de vista de que existe una falta de responsabilidad por parte del planificador urbano debido a que desde la ejecución del proyecto urbano-arquitectónico existe una desatención por parte del planificador urbano. "En sentido, la planificación (en sus diversas escalas) resulta ser el elemento preponderante a depurar, y es que sin duda una de las grandes cualidades del diseñador urbano es que aún sin que todavía se haya materializado la obra ya puede éste visualizarla mentalmente, incluso recorrerla y por consiguiente resultaría indudable lograr reconocer que se pudieran generaran en los nodos de cruce este tipo de fragmentos. Sin embargo, el recorrido virtual que realiza este creador de espacios no hace por lo general una pausa para reflexionar en torno a una propuesta que brinde una solución ante estas partes o porciones derivadas, sino que por el contrario la planificación del proyecto primordial continua su ritmo de ejecución. Es por ello que es posible afirmar que este tipo de fragmentos urbanos, son resultado de una indiferencia creativa por parte del arquitecto, urbanista e ingeniero encargado de proyectar aquella obra urbano-pública de gran escala". (Curzio, 2008, p. 69) y por lo tanto sin lugar a dudas el remanente urbano es un fenómeno que resulta prevenible y corregible.

\section{METODOLOGÍA}

El diseño de esta investigación es de tipo mixto, vinculando los enfoques cualitativo y cuantitativo para responder la interrogante planteada, así mismo es una investigación transversal lo cual se refiere a que se trata de un estudio realizado en un tiempo específico, en este caso la recopilación de los casos prácticos de remanentes urbanos fue llevada a cabo en los meses de Enero y Febrero del año 2020.

En primer término, se determinó la ciudad que se pretendería analizar, en este caso se seleccionó la zona urbana de Mexicali, Baja California. Posteriormente se integraron 151 AGEB (Áreas geo-estadísticas básicas que fueron obtenidas de INEGI) y de esa manera se conformó un gran polígono equivalente al área urbana de la ciudad de Mexicali y que represento una gran área de estudio equivalente a 7,435.83 hectáreas.

Figura 7. Mapa que muestra la integración de las 151 AGEB en la ciudad de Mexicali, Baja California

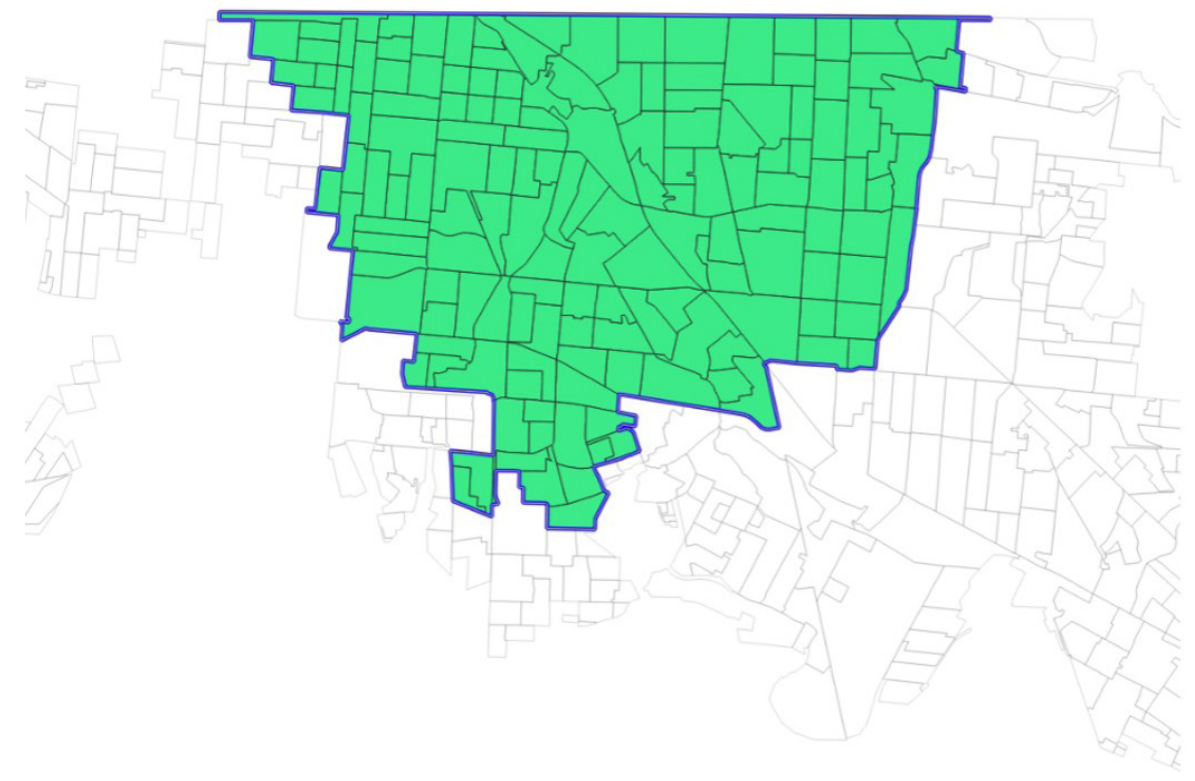

Fuente: Elaborado por los autores 
El criterio para la selección de las AGEBS estuvo en función de lograr establecer un gran polígono que resultara incluyente en cuanto lograr conjugar todas aquellas estructuras urbanas caracterizadas por tener continuidad (interconexión entre ellas) y que al mismo tiempo fueran zonas que estuvieran plenamente consolidadas, lo cual evito considerar zonas periurbanas que apenas se encuentran en vías de consolidación. (Ver Figura 8)

Posteriormente mediante la interpretación de Fotografías áreas ortogonales, se realizó un levantamiento digital localizando casos específicos (trazando polígonos individuales, identificando y dibujando el perímetro de los remanentes urbanos que fueron ubicados dentro del área de estudio). Paralelamente se realizó un levantamiento físico en campo para cotejar la información previamente obtenida en el levantamiento digital, así como fotografiar los casos más representativos.

Finalmente se integró la información empleando un Software especializado en sistemas de información geográfica (QGIS), con el objetivo de generar una base de datos con toda la información de los casos de estudio y paralelamente generar los mapas y estadísticas que fueron objeto de la presente investigación.

\section{RESULTADOS}

Una vez concluido el levantamiento de casos prácticos en la ciudad de Mexicali fue posible identificar 123 casos de remanentes urbanos que al momento de sumar su superficie representa un total de 29.20 hectáreas. Tomando como parámetro que el área de estudio tiene una superficie de 7,435.823 hectáreas, es posible afirmar que la superficie correspondiente a los remanentes urbanos representa un porcentaje del $0.393 \%$.

Después de revisar a detalle cada uno de los casos de estudios es posible señalar que contrastan las grandes diferencias que existen con lo que respecta a la superficie de cada remanente urbano. Específicamente el caso más pequeño (C-MX-013) cuenta con una superficie de $33.14 \mathrm{~m} 2$, mientras que el remanente urbano con la mayor superficie (C-MX-120) cuenta con 13,818.89m2. Así mismo cabe mencionar que la media (superficie promedio considerando los 123 casos) fue de 2,374.32 m2.

Figura 8. Fotografía aérea que muestra el área de estudio en la ciudad de Mexicali, Baja California

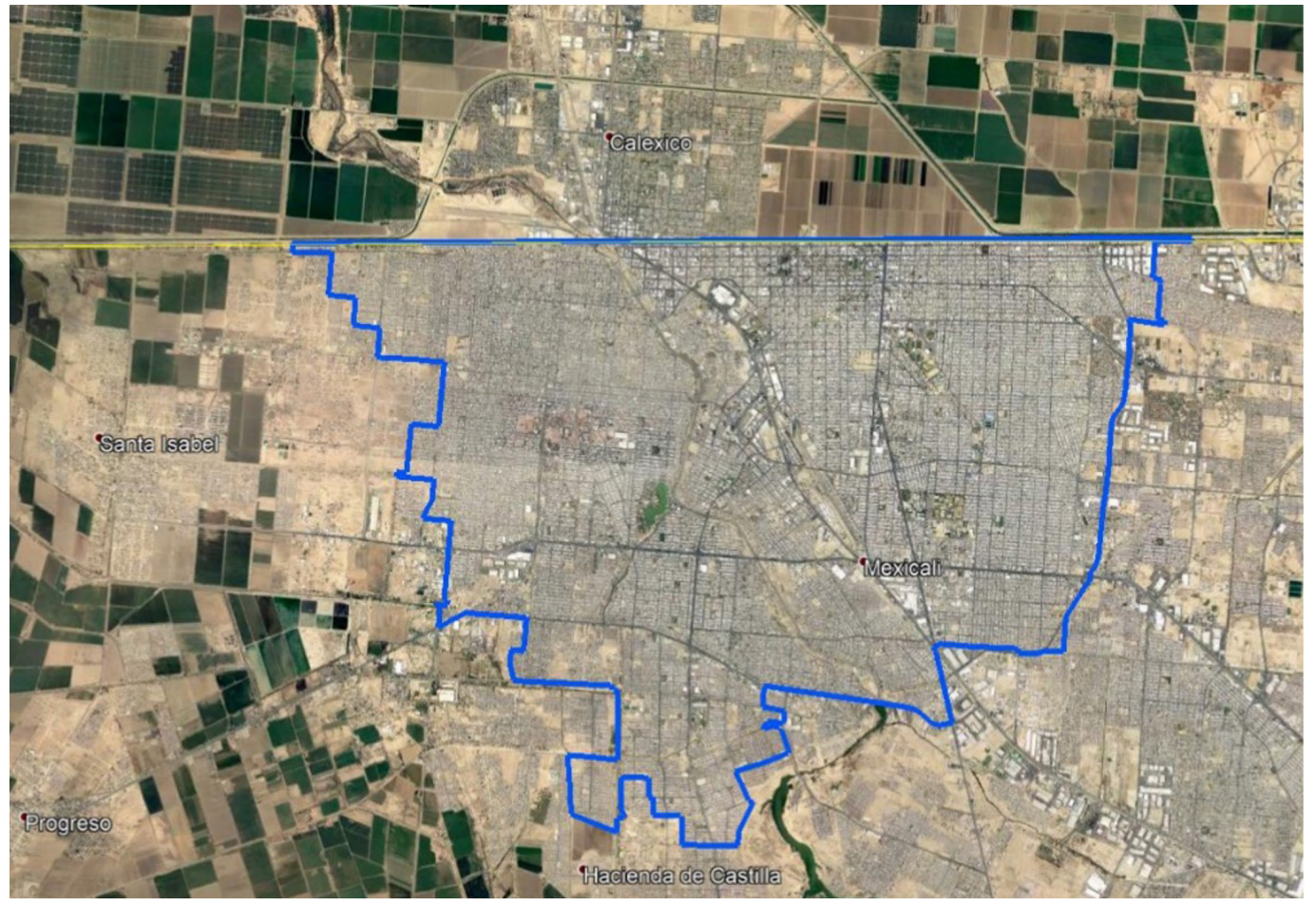

Fuente: Elaborada por los autores 
Figura 9. Tabla que muestra la superficie individual de los 123 casos de remanentes urbanos localizados en la ciudad de Mexicali, Baja California

\begin{tabular}{|l|c|c|c|c|c|c|}
\hline \multicolumn{7}{|c|}{ TABLA DE SUPERFICIES DE CASOS INDIVIDUALES (REMANENTES URBANOS) } \\
\hline CLAVE & AREA (m2) & CLAVE & AREA (m2) & & CLAVE & AREA (m2) \\
\hline
\end{tabular}

\begin{tabular}{|r|r|}
\hline C-MX-001 & 209.78 \\
\hline C-MX-002 & $2,948.55$ \\
\hline C-MX-003 & $4,540.41$ \\
\hline C-MX-004 & $8,097.88$ \\
\hline C-MX-005 & $11,699.68$ \\
\hline C-MX-006 & 234.38 \\
\hline C-MX-007 & 115.06 \\
\hline C-MX-008 & 107.29 \\
\hline C-MX-009 & $2,949.74$ \\
\hline C-MX-010 & $1,773.66$ \\
\hline C-MX-011 & 350.78 \\
\hline C-MX-012 & 106.97 \\
\hline C-MX-013 & 33.94 \\
\hline C-MX-014 & $2,824.76$ \\
\hline C-MX-015 & $3,117.69$ \\
\hline C-MX-016 & 834.99 \\
\hline C-MX-017 & 49.51 \\
\hline C-MX-018 & 167.89 \\
\hline C-MX-019 & $8,342.17$ \\
\hline C-MX-020 & $1,248.40$ \\
\hline C-MX-021 & $2,196.01$ \\
\hline C-MX-022 & $2,605.38$ \\
\hline C-MX-023 & 174.13 \\
\hline C-MX-024 & $1,741.02$ \\
\hline C-MX-025 & $9,610.42$ \\
\hline C-MX-026 & $4,424.47$ \\
\hline C-MX-027 & $7,741.44$ \\
\hline C-MX-028 & 198.08 \\
\hline C-MX-029 & 70.20 \\
\hline C-MX-030 & 47.17 \\
\hline C-MX-031 & 155.07 \\
\hline C-MX-032 & $6,687.01$ \\
\hline C-MX-033 & $5,438.10$ \\
\hline C-MX-034 & 160.03 \\
\hline C-MX-035 & $7,037.49$ \\
\hline C-MX-036 & $1,546.98$ \\
\hline C-MX-037 & $1,414.69$ \\
\hline C-MX-038 & $5,214.05$ \\
\hline C-MX-039 & 326.28 \\
\hline C-MX-040 & 195.98 \\
\hline C-MX-041 & 81.56 \\
\hline C-MX-042 & 151.10 \\
\hline & \\
\hline
\end{tabular}

\begin{tabular}{|r|r|}
\hline C-MX-043 & 317.77 \\
\hline C-MX-044 & 65.21 \\
\hline C-MX-045 & 85.00 \\
\hline C-MX-046 & 195.13 \\
\hline C-MX-047 & 472.64 \\
\hline C-MX-048 & $4,380.26$ \\
\hline C-MX-049 & $3,980.27$ \\
\hline C-MX-050 & $3,479.84$ \\
\hline C-MX-051 & $2,649.61$ \\
\hline C-MX-052 & 163.44 \\
\hline C-MX-053 & 68.47 \\
\hline C-MX-054 & $2,952.57$ \\
\hline C-MX-055 & $1,170.28$ \\
\hline C-MX-056 & 631.58 \\
\hline C-MX-057 & $2,026.91$ \\
\hline C-MX-058 & $3,578.89$ \\
\hline C-MX-059 & 183.39 \\
\hline C-MX-060 & 358.42 \\
\hline C-MX-061 & $5,607.67$ \\
\hline C-MX-062 & $5,288.67$ \\
\hline C-MX-063 & $1,857.83$ \\
\hline C-MX-064 & 996.50 \\
\hline C-MX-065 & $3,102.15$ \\
\hline C-MX-066 & $1,063.23$ \\
\hline C-MX-067 & $3,067.72$ \\
\hline C-MX-068 & 741.01 \\
\hline C-MX-069 & 688.37 \\
\hline C-MX-070 & $5,074.52$ \\
\hline C-MX-071 & $3,161.81$ \\
\hline C-MX-072 & 210.44 \\
\hline C-MX-073 & $2,584.10$ \\
\hline C-MX-074 & $2,608.35$ \\
\hline C-MX-075 & $7,331.85$ \\
\hline C-MX-076 & $11,825.58$ \\
\hline C-MX-077 & $8,395.20$ \\
\hline C-MX-078 & $1,657.58$ \\
\hline C-MX-079 & $1,347.74$ \\
\hline C-MX-080 & $1,470.25$ \\
\hline C-MX-081 & $4,268.51$ \\
\hline C-MX-082 & 205.33 \\
\hline C-MX-083 & $1,314.16$ \\
\hline C-MX-084 & $4,733.13$ \\
\hline & \\
\hline
\end{tabular}

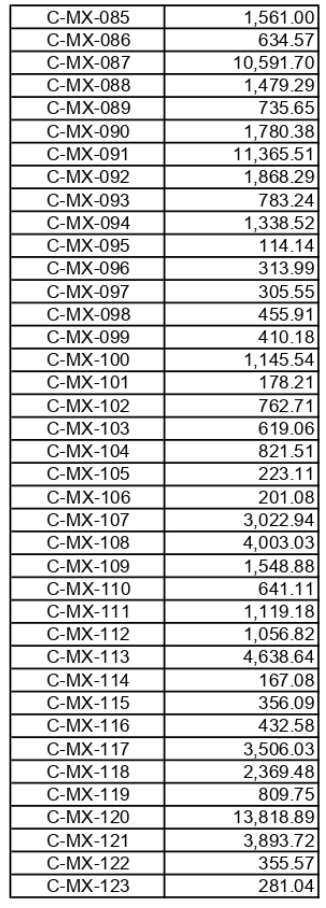

Superficie total $(\mathrm{m} 2) \quad 292,041.54$

Figura 10. Mapa que muestra el trazo y localización de los 123 casos de remanentes urbanos en la ciudad de Mexicali, Baja California

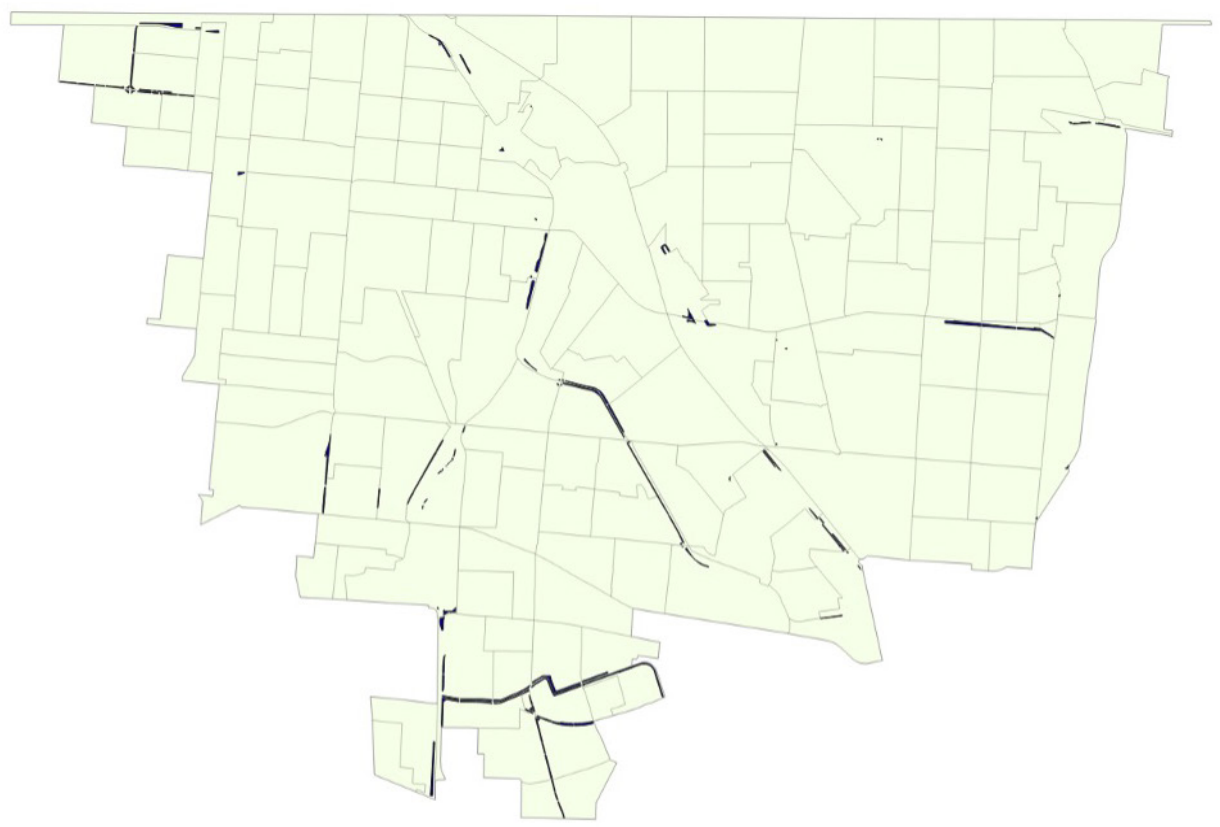

Fuente: Elaborados por los autores 


\section{PRINCIPALES HALLAZGOS}

A continuación se profundiza más el análisis mediante la exposición detallada de 5 casos específicos de remanentes urbanos (C-MX-014, C-MX-036, C-MX-040, C-MX-083 y C-MX085), concretamente dichos casos resultan ser algunos de los hallazgos más representativos que fueron detectados durante la investigación.

De manera específica en cada uno de dichos casos se realizará una exposición que incluye imágenes áreas de donde se localiza, así como fotografías individuales que ayudan a lograr comprender de mejor forma las características físicas de cada caso en particular; así mismo también se mencionan datos descriptivos relativos a la superficie y las coordenadas de localización.

\section{CASO: $C-M X-014$}

Superficie: $2,824.76$

Ubicación: Puente vehicular sobre Av.

Independencia (Colonia ex-ejido Zacatecas,

Mexicali, B.C.)

Coordenadas geográficas:

$32^{\circ} 38^{\prime} 10.34^{\prime \prime} \mathrm{N}-115^{\circ} 27^{\prime} 38.16^{\prime \prime O}$

Figura 11. Imagen área que muestra la ubicación del caso C-MX-014

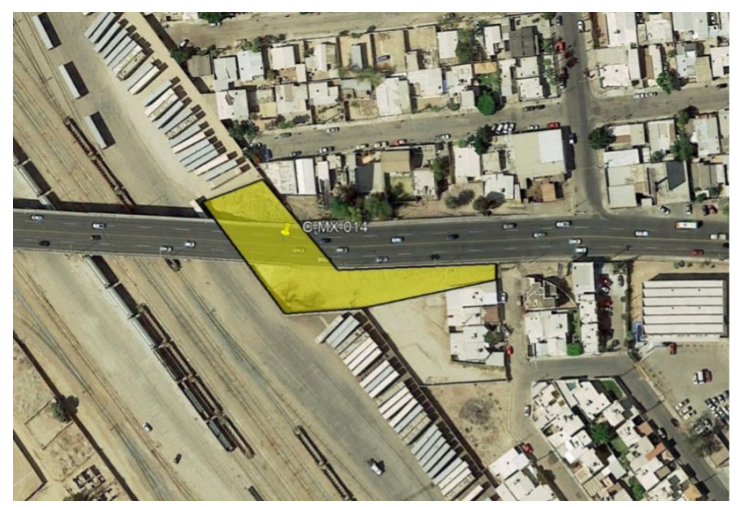

Fuente: Fotografía por los autores

El caso C-MX-014 es un notable ejemplo de un remanente urbano que surge a raíz de un proyecto de gran escala, surge como una resultante de la construcción de un nodo urbano (en este caso el puente vehicular ubicado en Av. Independencia que sirve para cruzar las vías del ferrocarril). Dicha obra dejo como consecuencia un par de áreas que quedaron sin resolverse, por una parte, se trata de un espacio olvidado (un intersticio) que quedo debajo de donde inicia la rampa del puente y por otra parte es posible observar un área lateral que también quedo olvidada y encerrada entre el límite de una propiedad privada y el costado del puente. (Ver Figuras 11 y 12)

Figura 12. Fotografía del caso C-MX-014

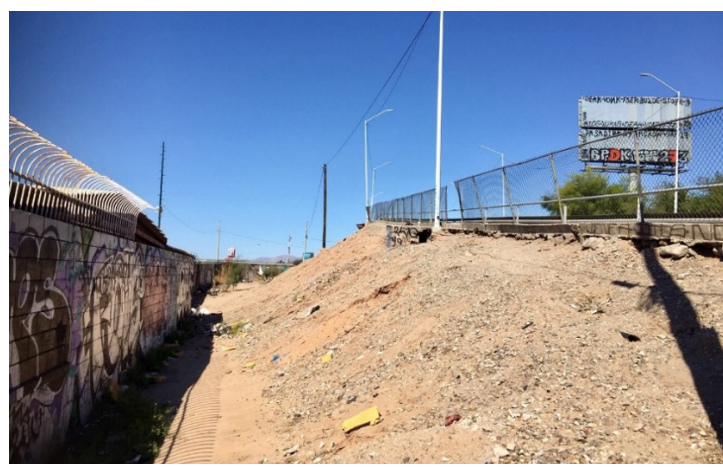

Fuente: Fotografia por los autores

Tomando en cuenta la ubicación, tamaño y las dificultades de accesibilidad resulta complejo pensar en alternativas de solución para este remanente urbano. En casos análogos la rehabilitación de este tipo de espacios se ha enfocado comúnmente con relación a contribuir en términos del paisaje urbano mediante áreas verdes. Sin embargo, esa solución pudiera no ser la indicada en este caso en particular debido a la limitada accesibilidad con que se cuenta, lo cual resultaría una problemática para cuestiones de mantenimiento en el cuidado de áreas verdes. De este modo pudiese encontrarse con una rehabilitación más acertada si se considerara construir un talud de concreto, al mismo tiempo que se considerara incluir una red de drenaje para canalizar las aguas pluviales que escurran por el talud.

\section{CASO: $C-M X-036$}

Superficie: $1,546.98 \mathrm{~m} 2$

Ubicación: Calle Centauro del Norte (Colonia División del Norte, Mexicali, B.C.)

Coordenadas geográficas:

$32^{\circ} 36^{\prime} 55.50^{\prime \prime} \mathrm{N}-115^{\circ} 29^{\prime} 55.68^{\prime \prime} \mathrm{O}$

Con lo que respecta al caso C-MX-036 es posible observar que se trata claramente de un derecho de vía que probablemente fue planeado con el objetivo de tener la posibilidad de extender la anchura de la calle en algún futuro. Sin embargo, parece complicado que esto pueda ocurrir debido a que dicho derecho de vía se va reduciendo a lo 
largo de dicha calle hasta el punto de desaparecer (inclusive esta situación también queda de manifiesto si se analiza a detalle la morfología del perímetro del caso de estudio, debido a que inicia con una anchura de $8.00 \mathrm{mts}$ y sin embargo el polígono termina con una anchura de $6.20 \mathrm{mts}$ ). (Ver Figuras 13 y 14)

Figura 13. Imagen área que muestra la ubicación del caso C-MX-036

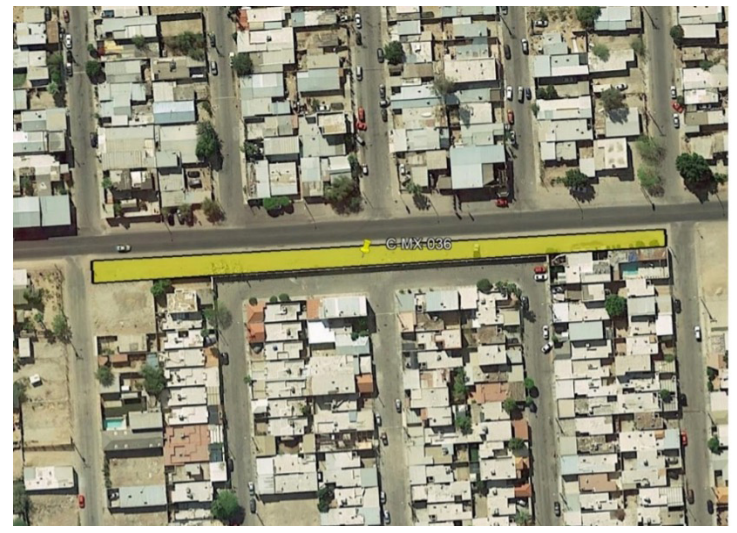

Fuente: Fotografía por los autores

Teniendo en cuenta las dimensiones de este remanente urbano (aproximadamente $212 \mathrm{mts}$ de largo y una anchura promedio de $7.10 \mathrm{mts}$ ) y por otra parte considerando que la calle donde se localiza este caso en particular no es una vialidad de alta velocidad, es posible asegurar que existen condiciones para poder diversas opciones de rehabilitación urbana (se podrían proponer áreas verdes con distintos enfoques que pueden incluir por ejemplo un carril para trotar o bien una ciclopista) así como áreas de descanso; básicamente el polígono completo podría transformarse en un parque municipal.

Figura 14. Fotografía del caso C-MX-036

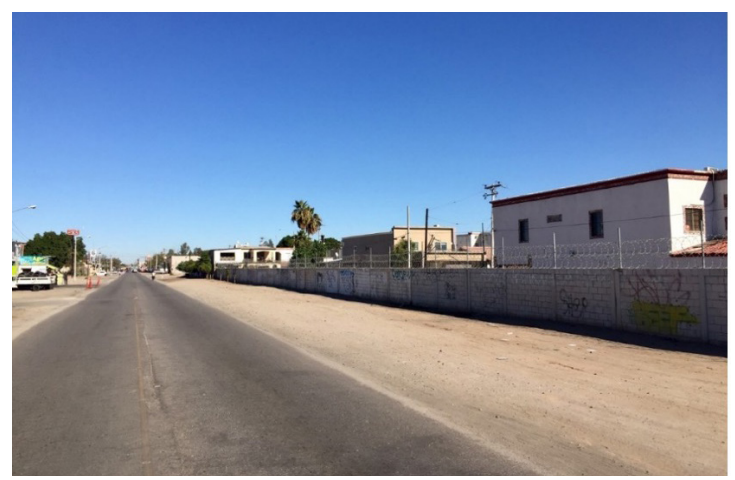

Fuente: Fotografía por los autores

\section{CASO: $C-M X-040$}

Superficie: $195.98 \mathrm{~m} 2$

Ubicación: Av. Eje Central (Colonia Santa

Monica, Mexicali, B.C.)

Coordenadas geográficas:

$32^{\circ} 36^{\prime} 53.55^{\prime \prime} \mathrm{N}-115^{\circ} 29^{\prime} 36.26^{\prime \prime} \mathrm{O}$

En el caso C-MX-040 es posible observar que se trata de un remanente urbano que surge como resultado de la construcción de la avenida Eje Central, en primera instancia se trata de un derecho de vía que existía (previamente a la construcción de dicha avenida) y posteriormente después de concluida la obra, fue posible observar que surgió un espacio intermedio entre el límite de las propiedades privadas y el inicio de la nueva avenida. Tomando en cuenta los anteriores antecedentes y además considerando las características físicas de dicho remanente urbano, es posible concluir que se este caso debe ser considerado como un intersticio. (Ver Figuras 15 y 16)

Figura 15. Imagen área que muestra la ubicación del caso C-MX-040

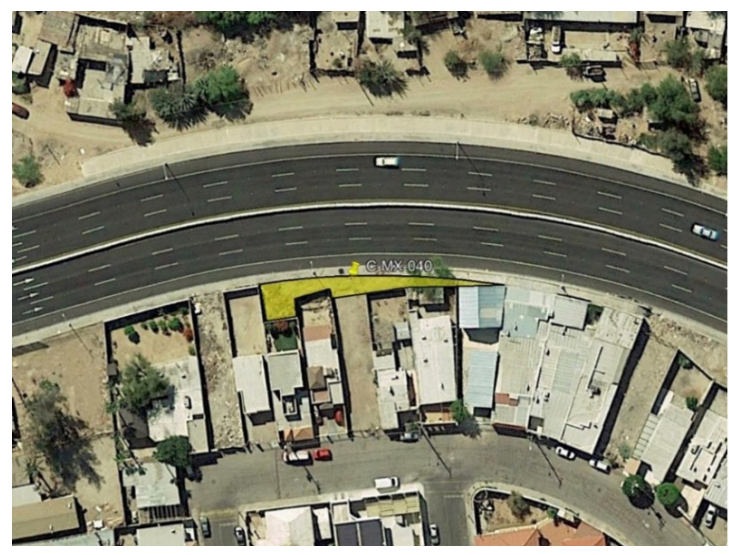

Figura 16. Fotografía del caso C-MX-040

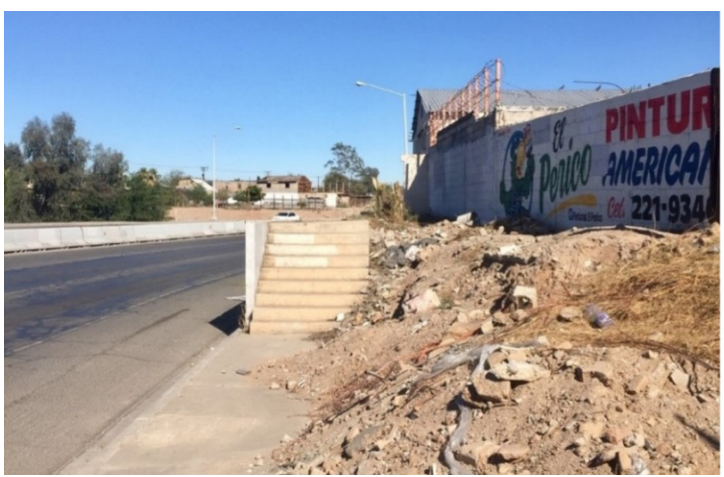

Fuente: Fotografias por los autores 
Por otra parte, considerando la reducida escala de este caso en particular y también teniendo en cuenta que no es una zona muy transitada por el peatón (la avenida Eje Central es de alta velocidad) se recomienda que la rehabilitación urbana debiese estar enfocada en poder brindar una solución en términos del paisaje urbano, básicamente se trata de realizar una limpieza y nivelación de la superficie con el objetivo de posteriormente colocar vegetación adecuada a las condiciones de la zona.

\section{CASO: $C-M X-083$}

Superficie: $1,314.16 \mathrm{~m} 2$

Ubicación: Calle lago Coyoatlan (Colonia Xochimilco, Mexicali, B.C.)

Coordenadas geográficas:

$32^{\circ} 36^{\prime} 28.23^{\prime \prime} \mathrm{N}-115^{\circ} 26^{\prime} 33.19^{\prime \prime O}$

Con lo que respecta al caso C-MX-083 se trata de un derecho de vía que corresponde a la infraestructura eléctrica de la ciudad, se trata de torres y líneas con cableado eléctrico que corren de forma paralela a la calle Lago Coyoatlan. Concretamente este remanente urbano posee una geometría de tipo rectangular y es posible observar que en una parte del perímetro si cuenta con una guarnición para delimitar el inicio de la calle sin embargo en el lado opuesto no existe ningún tipo de borde o limite, lo cual vuelve confusa la delimitación física en esa parte de la calle. (Ver Figuras 17 y 18)

Figura 17. Imagen área que muestra la ubicación del caso C-MX-083

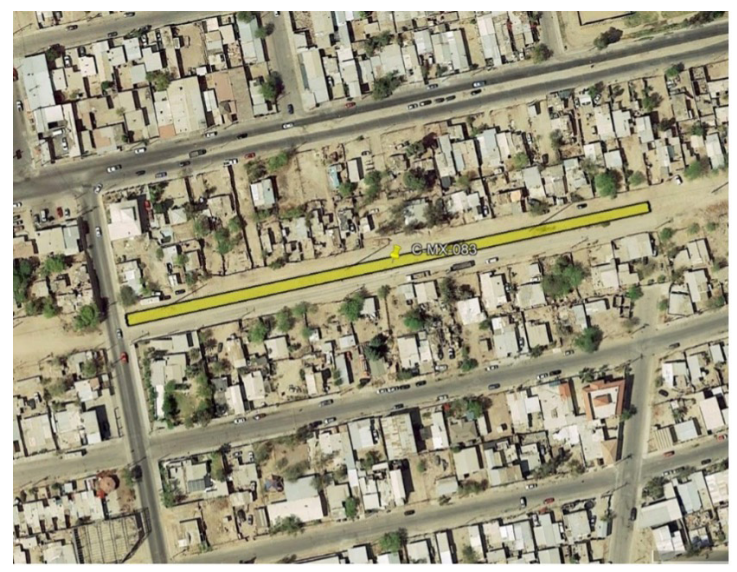

Fuente: Fotografía por los autores
De forma específica la rehabilitación de este tipo de remanentes urbanos parece ser bastante clara y $\sin$ muchas alternativas. Normalmente debido a la limitante de poder edificar debajo de las líneas eléctricas este tipo de espacios se resuelven mediante la implementación de áreas verdes y en este caso en particular habría también que pensar en construir una guarnición (borde) perimetral con el objetivo de establecer de manera clara los límites entre la calle y el remanente urbano.

\section{Figura 18. Fotografía del caso C-MX-083}

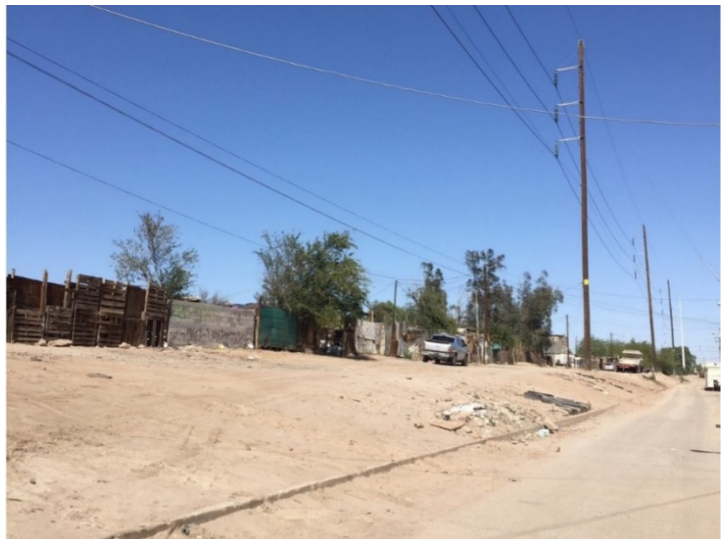

Fuente: Fotografía por los autores

\section{CASO: $C-M X-085$}

Superficie: $1,561.00 \mathrm{~m} 2$

Ubicación: Cruce de Calle San Miguel el Grande y vías del ferrocarril (Colonia Villa Mediterránea, Mexicali, B.C.)

Coordenadas geográficas:

$32^{\circ} 37^{\prime} 25.72 " \mathrm{~N}-115^{\circ} 27^{\prime} 9.26^{\prime \prime} \mathrm{O}$

Finalmente, al analizar este último caso de estudio (C-MX-085) es posible mencionar que se trata de uno de los tipos más comunes de remanentes urbanos que pueden encontrarse en distintas ciudades en el mundo, se trata de derechos de vía que se localizan paralelamente en los costados de las vías férreas que generalmente van atravesando de forma lineal las estructuras urbanas. En este caso el remante urbano se ubica en uno de los principales nodos de la ciudad (es el punto donde convergen las Avenidas Lázaro Cárdenas y Adolfo Lopez Mateos).y cuenta aproximadamente con $227 \mathrm{mts}$. de longitud, teniendo en cualquier punto de su perímetro una amplia facilidad de acceso (no existe ninguna barrera física). (Ver Figuras 19 y 20 p. 83) 
Figura 19. Imagen área que muestra la ubicación del caso C-MX-085

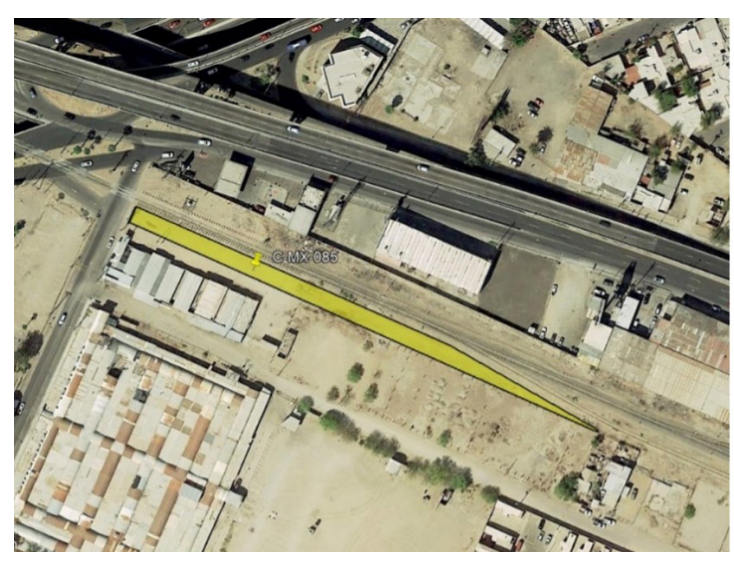

Figura 20. Fotografía del caso C-MX-085

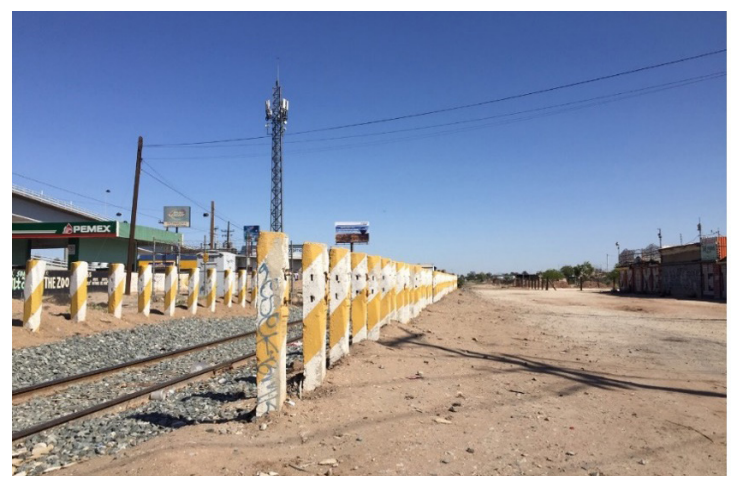

Fuente: Fotografias por los autores

Si bien es cierto que este remanente urbano no presenta grandes muestras de marginación urbana (la superficie esta nivelada y limpia, libre de desperdicios) si se podría pensar en llevar a cabo algún tipo de implementación urbana a nivel paisajístico, frecuentemente este tipo de derechos de vía (sobretodo los que encuentran plenamente enclavados en los núcleos urbanos de las ciudades) pueden aprovecharse como áreas de recreación para los habitantes, en ocasiones se han implementado corredores verdes que cuentan con pistas para trotar o para circular en bicicleta.

\section{CONCLUSIONES}

Los conflictos propios de la naturaleza humana provenientes de su deseo de posesión e instinto territorial, la han motivado a delimitar su territorio mediante señales y actitudes o huellas fisiológicas como desechos orgánicos sólidos, líquidos y olfativos o de una forma más elaborada podemos pensar en elementos artificiales como bardas, líneas, muros, enrejados, cercas, empalizadas, ramas, objetos y materiales reutilizables, siendo los principios de la apropiación del espacio territorial, sin tener todavía en cuenta una delimitación dimensional del territorio.

Sin embargo, la distribución territorial de las capacidades y bondades naturales no es homogénea y no se ha prodigado democráticamente a lo largo y ancho de las grandes extensiones de la superficie terrestre: surge la necesidad de desplazamiento de un lugar a otro para obtener los satisfactores de supervivencia nómada. Los impulsos de movilidad perfilaron los caminos para los primeros asentamientos humanos sedentarios que dan cuenta del proceso en que, al tener bienes y productos en demasía unos y faltantes otros, surge el intercambio comercial con las dificultades inherentes a quienes poseen mucho, poco o nada.

La producción artificial masiva de satisfactores dio inicio a las actividades industriales a través de procesos en serie reforzados y auxiliados por máquinas y equipo que incrementó en cantidad y calidad los objetos manufacturados, bajo una premisa el dimensionamiento espacial del territorio, fortaleciendo los espacios sociales, laborales, comerciales e industriales y habitacionales con un consumo recurrente de territorio dentro de los núcleos urbanos, para dar mayor certeza y legalidad a estas actividades económicas, nace el Estado como órgano regulador de las condiciones, lugares y estrategias de desarrollo en los sectores agrícola, minero, urbano e industrial, motorizadas tanto por el sector público como el privado.

Así varias ciudades se transforman con el paso del tiempo determinando sus aspectos jurídicos, sociales, culturales, económicos, de desarrollo urbano y los derechos de propiedad como capital que descansa en un régimen de propiedad, en obras de urbanización como medios de producción, fuerzas productivas y relaciones de producción; las modalidades en que se desenvuelven las actividades económicas establecidas en zonas, áreas o sectores de ciudades son revisadas desde la óptica del cumplimiento de la normativa aplicable a los espacios aptos y no aptos para el desarrollo urbano y la protección de los recursos naturales disponibles.

Derivado de la presente investigación es posible mencionar que la ciudad de Mexicali mantiene 123 remanentes urbanos en una fase de 
gestión y valoración del territorio, reflejando el proceso de evaluación de las transformaciones productivas y las dinámicas territoriales que ha experimentado en su diferentes etapas de crecimiento urbano y su implementación de las diversas actividades económicas, con una visión estratégica promoviendo las reservas de suelo aptas para el emplazamiento de una diversidad de opciones para su disposición de ocupación, contando con diferentes morfologías de remanentes urbanos que conducen a generalizar situaciones que mantienen tramos vulnerables, espacios perdidos, espacios con potencial de recuperación, espacios inactivos sin una definición clara de los coeficientes de ocupación y utilización del suelo, es decir, una producción urbana dispersa y desordenada.

La ciudad de Mexicali debe tomar más en consideración los espacios residuales existentes determinando el control y las disposiciones normativas y los atributos, características y potencialidades de la tierra ruraly el suelourbano, como factores que inciden en la posibilidad de desarrollar socioeconómicamente territorios y una mejor cohesión social del tejido urbano existente; estando regulados concurrentemente bajo los tres órdenes de gobierno a través de la normativa que administra los derechos de propiedad inmobiliaria.

En el orden municipal la regulación se ve materializada, en un primer momento, con el documento denominado "Constancia de Alineamiento y Compatibilidad Urbanística", el cual señala tácita y explícitamente, de acuerdo con planes y programas de desarrollo urbano vigentes, las condicionantes y regulaciones que administran la amplísima gama de modalidades ejercidas en la utilización o usos del suelo viables para la consolidación de objetivos y propósitos propuestos para una mejor, óptima y sustentable calidad de vida.

La interacción coordinada de los órdenes de gobierno, define un espacio social multifactorial disponiendo un alineamiento y derechos de vías determinando una delimitación dimensional del territorio. Por lo tanto las distintas manifestaciones de espacios residuales son la expresión de la lectura espacial del anti-espacio analizando la subutilización de espacios debe ser el punto de partida de una política de desarrollo urbano con la premisa esencial de reconstruir el tejido social de la ciudad de Mexicali y pormedio de las acciones conjuntas entre los diferentes niveles de gobierno, la iniciativa privada y la sociedad creara las oportunidades de intervención necesaria de estos 123 remanentes urbanos.

Reconociendo la existencia de espacios públicos que se encuentran muy deteriorados sin contar con áreas verdes y con problemas de tipo social y casas abandonadas y la falta de servicios en algunas zonas de la ciudad, es oportuno sobre todo la participación municipal en el fortalecimiento de áreas públicas y de una mayor atribución a la reconstrucción del tejido urbano social, el apoyo de las instancias recaudadoras para coordinar el pago del impuesto predial y derechos de las viviendas recuperadas y por supuesto contar con las facilidades necesarias para la obtención de licencias referentes a las intervenciones físicas que se realicen en cualquier polígono territorial de los espacios remanentes. C

\section{REFERENCIAS BIBLIOGRÁFICAS}

Berruete, F. (2015). Vacios urbanos en la ciudad de Zaragoza (1975-2010) Oportunidades para la estructuración y continuidad urbana. (Tesis Doctoral). Universidad Politécnica de Madrid, Madrid-España.

Berruete, F. (2017). Los vacíos urbanos: Una nueva definición. Revista URBANO, 35, 114-122.

Cléments, G. (2007). Manifiesto del Tercer Paisaje. Barcelona-España: Gustavo Gili.

Curzio, C. (2008). El origen y las características de los fragmentos urbano-públicos residuales. In Cuadernos Geograficos (Vol. 42). Granada-España: Universidad de Granada.

Doreste, A. (2011). Intersticios urbanos: reflexiones a partir de un caos. In CIUDADES (IM) PROPIAS: La tensión entre lo global y lo local (pp. 273 - 284). Valencia-España: CIAE - Universitat Politècnica de València.

Díaz Cruz, N. A. (2015). Análisis del paisaje residual en Bogotá. (Magíster en Geografía). Universidad Nacional de Colombia, BogotaColombia.

García Bellido, J., \& Gonzalez Tamarit, L. (1979). Para comprender la ciudad: Claves sobre los procesos de producción del espacio. Madrid-España: Nuestra Cultura.

Hall, P. (1996). Ciudades del mañana. BarcelonaEspaña: Ediciones Del Serval.

Hannequart, J. P. (1993). Le Droit European des dechets. Bruselas - Belgica: Inst. INEGI. (2010). www.inegi.org.mx. 
Maciocco, G. (2008). Fundamental Trends in City Development. United Kingdom: Springer.

Nefs, M.(2006). Unused urban space: Conservation or transformation? Polemics about the future of urban wastelands and abandoned buildings. City \& Time, 2, 47-58.

Padilla y Sotelo, L., \& Juárez, M. d. C. (2000). La dimención espacial del crecimiento poblacional de Mexicali. Investigaciones Geográficas, 43, 88 - 104.

Rosenau, H. (1986). La ciudad ideal: Su evolución arquitectónica en Europa. Madrid-España: Editorial Alianza.

Vizcarra, B. (2019). Despoblamiento en barrios fundacionales fronterizos: el caso de Pueblo Nuevo en Mexicali, México. INTERSTICIOS SOCIALES, 18, 303 - 325.

Zarate Martin, A. (2003). El espacio interior de la ciudad. Madrid - España: Editorial Sintesis. 